\title{
Efficient Switched Services over a DVB-SH/3G Network
}

\author{
A.JEDIDI, M.TLAIS and F.WEIS \\ INRIA/IRISA-Rennes, France \\ azza.jedidi@irisa.fr
}

\author{
Sylvaine KERBOEUF \\ Alcatel Lucent Bell Labs, France \\ sylvaine.kerboeuf@alcatel-lucent.fr
}

Permission to make digital or hard copies of all or part of this work for personal or classroom use is granted without fee provided that copies are not made or distributed for profit or commercial advantage and that copies bear this notice and the full citation on the first page. To copy otherwise, or republish, to post on servers or to redistribute to lists, requires prior specific permission and/or a fee. Mobimedia'09, September 7-9, 2009, London, UK. Copyright 2009 ICST 978-963-9799-62-2/00/0004 ... \$5.00

\begin{abstract}
Fourth generation networks result from the cooperation between different existing networks, thus taking benefit from their complementary properties. In this context, recent researches have addressed Digital Video Broadcast (DVB) networks, which benefit of very high broadcast capacities, but are unidirectionnal. Coupling a DVB network with a bidirectional network brings bidirectionality and service interactivity.

In this context, we focus on a DVB-SH (satellite services for hand-held devices) network, which is a hybrid (satellite/terrestrial) standard. We couple DVB-SH with a 3G cellular network, thus providing bidirectionality. DVB-SH benefits from a very high bandwidth capacity that allows unidirectional IP-TV channels broadcast.

A residual bandwidth in the DVB-SH path may still be available because of the variable bit rates of served flows.

In a previous study [1], we focused on this small residual bandwidth and its potential utilization. Our idea was to use this architecture in order to provide interactive low cost services over DVB networks. We specified an efficient switching of IP services, coming from $3 \mathrm{G}$ networks, to the residual bandwidth of DVB networks.

In this paper, we extend DVB residual bandwidth thanks to some DVB channels reserved by the $3 \mathrm{G}$ provider and study the impact of this extension on the type and the quality of targeted services. This paper particularly highlights the scheduling algorithm of the 3G flows switched over DVB residual bandwidth. It also presents a user subscription pre-
\end{abstract}

diction algorithm that aims to guarantee efficient switching while maintaining service continuity. ${ }^{1}$

\section{Keywords}

Heterogeneous wireless networks, DVB, 3G, residual bandwidth, network convergence, multimedia, low cost services, mobile TV, quality of experience

\section{INTRODUCTION}

Recently, a growing interest has been shown in multimedia networking mainly due to the emergence of efficient audio/video encoding techniques and the proliferation of enhanced audio-visual services. The demand for these kinds of applications has quickly increased. Major advances in communication and network technologies have made multimedia services technically and economically feasible in any type of environment. Digital TV and multicast IP represent the best processes to deliver multimedia content respectively through broadcast and Internet-based networks. Regarding broadcasting standards, Digital Video Broadcasting (DVB) [2] is expected to be the prominent European television broadcast standard for the next decades, as well through a satellite-based technology (DVB-S) [3], as in terrestrial television (DVB-T) [4], cable (DVB-C) [3] or for hand-held devices (DVB-H) [5]. The DVB technology provides relatively high bandwidth data channels but based on uni-directionality, thus neglecting interactivity.

DVB-SH [6], satellite services for hand-held devices, is a hybrid (satellite/terrestrial) standard. It is defined as a system for IP based media content and data delivery for hand-held terminals, via satellite. Satellite transmission guarantees wide area coverage. Moreover, it is coupled with terrestrial gap fillers assuring service continuity in areas where the satellite signal cannot be received (built-up areas for example). DVB-SH provides users with a variety of services, which could be classified in several categories. It offers realtime applications. Examples are TV-like broadcasting, live broadcasting and notification, which consists in broadcast notifications sent according to the preferences of the user (notifying a football fan of the retransmission of his preferred team matches for instance) and games, like real-time quizzes or multiplayer online role-playing games, etc. It also provides applications to download. For large general audiences, data file purchase services are offered, either on a subscription basis, such as downloading every morning the

\footnotetext{
${ }^{1}$ This paper presents results from a collaboration between INRIA ACES research team and the MAG project from Alcatel-Lucent Bell Labs
} 
electronic version of the user's newspaper, or on an impulsive purchase basis, like for films, books and audio CD purchase.

Besides, one of the main characteristics of the Internet

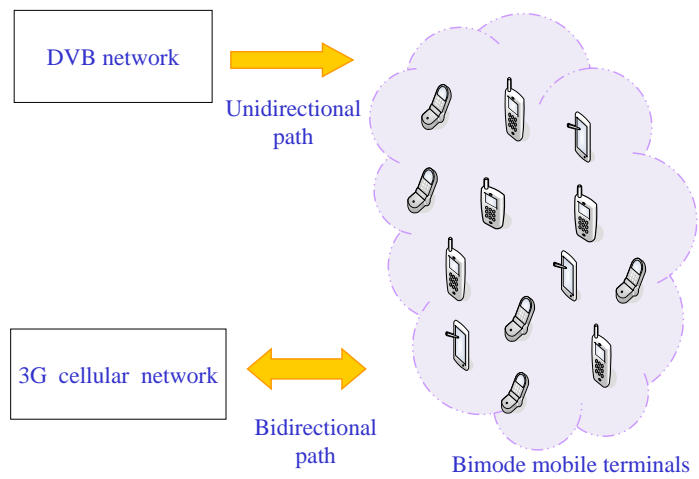

Figure 1: Coupling DVB with 3G network

world is its bidirectionality, permitting full interactivity to users. In the context of our study, a DVB-SH broadcast network is combined with a third generation cellular network (3G network) to ensure this bidirectionality, as shown in Figure 1. Actually, this convergence takes benefit from 3G and DVB networks. 3G network characteristics, especially upload link, enable added-value services and applications that are interactive and more personalized. DVB-SH benefits from an expensive but very high bandwidth capacity that allows unidirectional IP-TV channels broadcast. A residual bandwidth in the DVB-SH path may still be available because of the variable bit rates of served flows as shown in Figure 2.

In a first step [1], we focused on this small residual bandwidth, initially unused. We proved through simulations that thanks to this small bandwidth several low cost services can be offered to a large group of users, which was not possible using only $3 \mathrm{G}$ networks. The idea was to realize an efficient switching of IP data, coming from $3 \mathrm{G}$ networks, to the residual bandwidth of DVB networks. This scenario occurs especially when $3 \mathrm{G}$ contents or services become very popular. Thus their transmission may take benefit from the large broadcasting capacities offered by DVB-SH.

The 3G switched flows were inserted over DVB-SH residual bandwidth in a First In First Out mode.

In this paper, we bring two major improvements. First, we refine our flow switching model through the definition of more adapted scheduling algorithms. Second, we extend DVB residual bandwidth. Actually, $3 \mathrm{G}$ provider can pay for reserving some DVB channels, as shown in figure 3.

Our objective is to study the impact of this extension on the type and the quality of targeted services. In this context, our study presents a user subscription prediction algorithm that aims to guarantee switching efficiency while maintaining service continuity.

The next section presents related work to DVB/3G network inter-working. Section 3 describes the system architecture that will be used in this work. Section 4 presents the service scenario. Section 5 and section 6 address our proposals

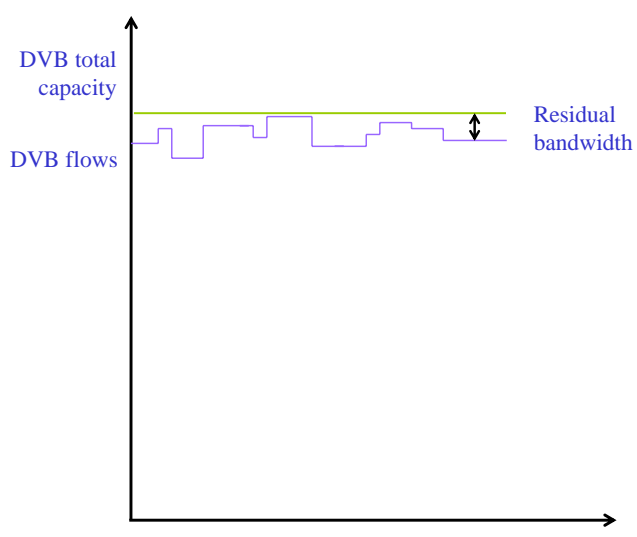

Figure 2: Residual bandwidth

to switch IP data to DVB networks, while treating related problems. Simulation results for the proposed solutions are shown. Finally, section 7 draws our conclusions.

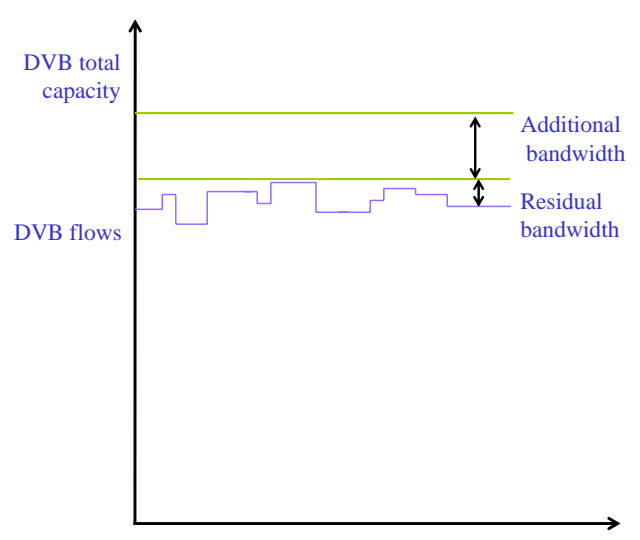

Figure 3: Extended residual bandwidth

\section{RELATED WORK}

Several works have addressed the coupling of DVB with another network, in order to achieve bidirectional channels. The main goal of those works was to enable users to interactively communicate with the network using the fixed line and mobile phone.

For instance, the study [7] discussed the coupling of DVBC/DVB-T with a satellite return channel and terrestrial systems as ADSL, ATM. The goal was to achieve interactive services with users participation.

The ATHENA European Research project [9] has proposed a specific structure of hybrid communications access network, exploiting the particularities of the DVB-T system in offering broadband connectivity to INTERNET for a large category of users. In the context of ATHENA project, the authors in [10] have proposed to manage the bandwidth between IP data and DVB-T flows. The solution is based on 
two bandwidth management systems, respectively for DVB$\mathrm{T}$ and IP, inter-working together in order to perform specific improving tasks on the corresponding flows.

In [8], the authors have studied the coupling of DVB-H/DVB$\mathrm{T}$ with a cellular network. The goal was to use bidirectional channels in order to improve the handover between a DVB network and another network. Thus, the lost data during the handover have been recovered through the up-link channels.

In our work, we are going further in inter-working DVB$\mathrm{SH}$ and mobile IP networks. We address several aspects of switching data to DVB networks, mainly: services that may be proposed to utilize the DVB residual bandwidth, switching decision to look if a network switch is useful or not, IP data insertion to insert flows coming from the $3 \mathrm{G}$ network in the DVB network, flow scheduling to optimize the use of the residual bandwidth and prediction of user subscription to guarantee that a switch does not affect the user experience.

\section{PROPOSED ARCHITECTURE}

The architecture studied in the scope of this work is based on an unidirectional DVB-SH broadcast network, coupled with a third generation cellular network. We concentrate our efforts on innovative low cost services that may be brought by non real time flows while efficiently using the DVB residual bandwidth. Figure 1 has shown a simple DVB-3G architecture in which $3 \mathrm{G}$ and DVB paths are completely separated. A more realistic coupling architecture takes into account that $3 \mathrm{G}$ and DVB paths may be linked through some entities to provide a continuous service. Hence, a new entity is needed to switch IP data from $3 \mathrm{G}$ to DVB, and then to insert these data within DVB residual bandwidth. We introduce a device called unicast-broadcast router (UBR) that manages the interface with the service provider. It is mainly responsible for inserting IP data switched from $3 \mathrm{G}$ network in the DVB-SH network.

Figure 4 details the architecture elements. The content cre-

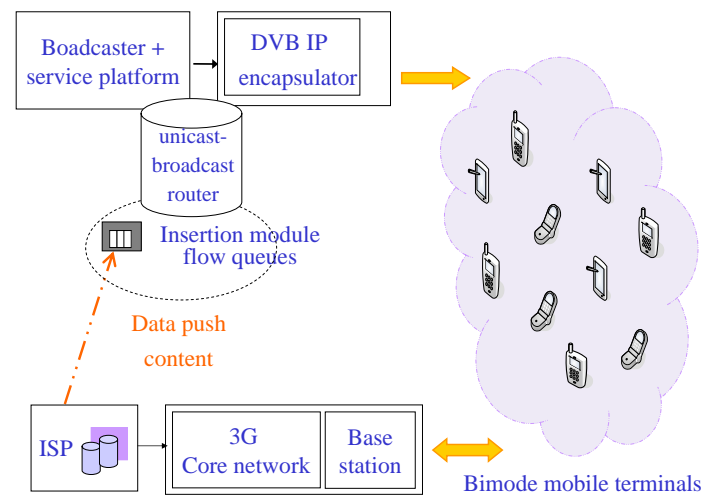

Figure 4: IP data switch

ation is on the broadcaster/service platforms in the DVB-SH network side, and the $\mathrm{ASP}^{2}$ is on the $\mathrm{ISP}^{3}$ on the $3 \mathrm{G}$ network side. Both, they are responsible for content creation and service application. They feed terminals with content encoded in the appropriate format, via streaming, download, or file carousel delivery. Moreover, they generate service description metadata. The DVB-SH broadcast network is not only responsible for video flow broadcasting, but it also takes into account IP flows that need to be encapsulated before being broadcasted. The $3 \mathrm{G}$ network permits bidirectional transmission of IP data, thus providing interactive and personalized services. The terminal is the user device, which acquires and consumes the received content. It is bimode, i.e. it has two network interfaces: $3 \mathrm{G}$ and DVB-SH. The considered terminals have a random mobility in the network. The IP encapsulator manages IP flow encapsulation on MPEG 2-TS packets; it handles time slicing and error correction mechanisms.

\section{SERVICE SCENARIO DESCRIPTION}

Users subscribe to services via the $3 \mathrm{G}$ network. The latter reserves a unicast channel for each user. The more users' requests, the more unicast channels. Thus, based on the number of requests, it might be interesting to switch from multiple unicast channels to the residual bandwidth of the DVB network. The initial idea behind this use case is to design low cost services, which occupy the initially not used DVB bandwidth. Hence, a component in the $3 \mathrm{G}$ infrastructure, at the ASP level of the architecture, stores and manages the number of subscription. Then, the flows are queued and asynchronously inserted in the DVB-SH network as shown in Figure 4.

This paper is focused on $3 \mathrm{G}$ scheduled services. The availability of these services is announced in advance, so that users can subscribe before the service start. Thus, the number of subscriptions to a $3 \mathrm{G}$ scheduled service is known before it starts.

Very popular services lead to deploying a huge number of unicast $3 \mathrm{G}$ channels. Hence, it becomes interesting to switch the service over the residual DVB-SH broadcast channel instead of deploying as many unicast channels. Obviously, the delivery of the switched services will be delayed as they are transmitted over a very small bandwidth. Nevertheless, we achieve a valuable gain as we avoid to charge our 3G network with many unicast connections delivering the same content. The challenge is to guarantee, for these services, an acceptable user experience, especially in terms of transmission delays. This approach is interesting for videos of several minutes of sustainability; they may start several minutes later. Moreover, those services are offered at low costs to compensate the introduced delay of delivery. Targeted services are periodic video delivery of flash news like weather and traffic, asynchronous video delivery of commercial information as in supermarkets or downloading of popular softwares.

\subsection{Switching decision}

If a $3 \mathrm{G}$ scheduled service is very popular, the ISP server sends a notification message to the UBR router. This message contains the size of the file to be sent. Upon receipt of this message, the UBR router replies by a message that contains two fields: the estimated time before the ASP server

\footnotetext{
${ }^{2}$ Application Service Provider

${ }^{3}$ Internet Service Provider
} 
starts sending IP data, and the estimated time the file may spend in delivery. According to these parameters, the $3 \mathrm{G}$ server chooses either to switch to DVB-SH after the specified waiting time or to send data immediately through $3 \mathrm{G}$ networks.

\subsection{IP data insertion}

The design of IP flow insertion in the DVB-SH network involves multiple mechanisms and raises several problems. The main problem is how to optimize available bandwidth sharing between DVB-SH original services and the 3G contents transmitted over the DVB-SH path. In our context, a dynamic bandwidth allocation approach seems to be more adapted as it optimizes the use of available bandwidth while maintaining a guaranteed part for $3 \mathrm{G}$ services. Figure 5 shows our proposed system to insert IP data in DVB-SH networks. This system extends the classical one used by DVB-SH networks. It is based on many sub-queues connected to the output through several main queues. The first queue (higher one) is the most priority queue. It is first served to be emptied. Then, the priority decreases until the last queue is served. We distinguish three main queues, each of them manages several sub-queues. The first queue is reserved to deliver notifications (signalization). The second one is reserved for multimedia and real time flows. This queue manages several sub-queues according to the number of real time flows to be delivered. The third queue is reserved for non real time flows.

Our idea is to extend this system to build an algorithm

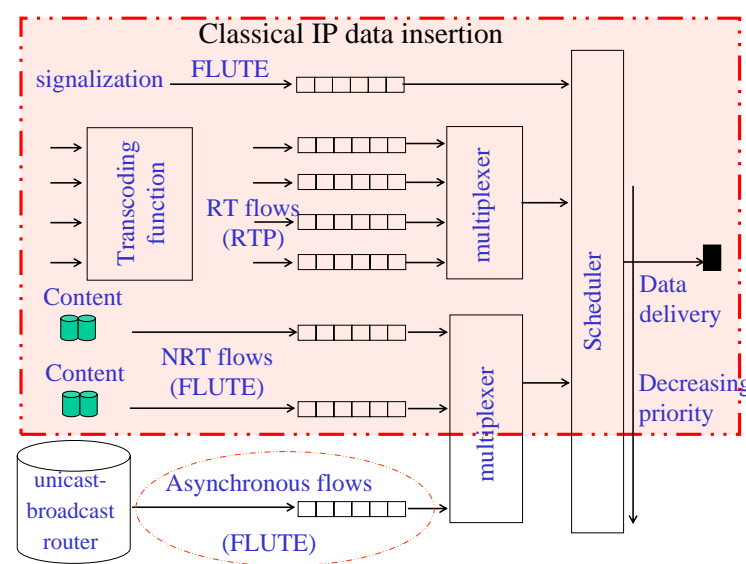

Figure 5: IP data insertion

that uses a pre-fetching approach for the switched services. Hence, for the insertion system, we propose to add a new sub-queue in the entry of the non real time queue, as shown in Figure 5. This sub-queue is reserved to IP data switched from $3 \mathrm{G}$ to DVB network. It is called asynchronous subqueue to reflect its asynchronous way to deliver data. It stores data in its buffer, then once a free bandwidth is available, it delivers data to users. When no more bandwidth can be freed, then no data are delivered. And so on, until all switched data are delivered or the waiting time the data may stay in the sub-queue is expired. In this case, it is possible to re-switch data to the $3 \mathrm{G}$ network to be delivered immediately.

The Unicast Broadcast Router receives the requests sent by application servers asking it to accept a switch from $3 \mathrm{G}$ to DVB channels. The UBR router uses a FIFO (first in first out) policy and estimates the waiting time that may be perceived by each application server before packets are delivered. Then, each application server decides if it is able to wait or not for the bandwidth availability.

\subsection{First results}

In [1], we have proved through simulations that, we can switch two or three popular IP scheduled services of about 5 megabits of content over DVB residual bandwidth, while maintaining acceptable transmission delays. The simulation parameter we used are detailed in section 5.1. We also have shown that delays become more important as the number of $3 \mathrm{G}$ switched services and the amount of delivered data increase.

Even if those services are delivered at very low costs, we should guarantee satisfying delivery delays to offer a good quality of experience.

Starting from this observation, we explore possible quality of service enhancements.

\section{EFFICIENT SCHEDULING OF THE SWITCHED SERVICES}

An important point is that the impact of delays on user experience depends on the service type. A software download may be delayed by two or three hours if it is offered at a very low price, but such a delay will not be acceptable for a car driver who asks for the traffic flash news, before starting his trip. Some services have more severe constraints, and should be broadcasted over the residual bandwidth before the others. This observation leads us to review our 3G switched flow scheduling policy. Popular 3G services, which are switched over the DVB path, have obviously lower priority than original DVB services. But, the scheduling of 3G popular services, asking simultaneously for being broadcasted over the residual DVB bandwidth, remains an important challenge. In our previous study [1], we simply used a First In First Out scheduling algorithm. Actually, the first service programmed to be played, was the first to ask for being switched over DVB, and thus was the first to be inserted in DVB residual bandwidth. The scheduled service playing date seems to be a fair criteria of scheduling, as it follows the chronological order of services. However, other criteria may be considered. For instance, it is possible to take into account the service type and sustainability. It is also possible to take into account the service financial cost.

The main objective of our work is to enhance $3 \mathrm{G}$ network performances as we avoid the deployment of many useless 3G unicast connections, delivering the same content. So, it seems relevant to privilege the transmission over DVB-SH of services with the greatest impact on $3 \mathrm{G}$ network performances. For instance, most popular $3 \mathrm{G}$ services necessitate more unicast $3 \mathrm{G}$ connections. Long duration $3 \mathrm{G}$ services use several unicast connections for longer durations. Such services should be the first ones to be broadcasted over the DVB-SH residual bandwidth.

At the UBR level, the first $3 \mathrm{G}$ queued service is transmitted immediately over the DVB-SH residual bandwidth. Then, the second one receives an estimation of the waiting time for 
being broadcasted and decides whether it accepts to wait or whether it refuses and delivers its content over the $3 \mathrm{G}$ path. Hence, the rank of the service in the queue is a key criteria to estimate the waiting time. The better this rank, the lower the estimated waiting time, thus increasing the probability for the $3 \mathrm{G}$ server to accept to be delayed.

Content size may be a relevant criteria of scheduling, as long contents monopolize 3G network capacities for longer durations. But, content size may also impact the service quality in another way. Actually, if we privilege the transmission of $3 \mathrm{G}$ popular services with smaller contents, we maximize the global number of broadcasted services.

\subsection{Simulation parameters}

Our simulations focus on $3 \mathrm{G}$ flow delivery over the DVBSH path. We evaluated several tools, and we finally chose to use OPNET Academic edition simulator [11] since it is more adapted for realistic large-scale scenarios. Using OPNET, we have simulated the functional entities of our architecture. Indeed, the implementation of many components was needed, for example DVB content creation, ASP application server, UBR router, DVB/3G networks and mobile terminals.

The implemented DVB-SH network offers a total bandwidth of $2615 \mathrm{kbits} / \mathrm{s}$. There are 10 DVB channels provided on this path, each channel is broadcasted at a variable bit rate of about $260 \mathrm{kbits} / \mathrm{s}$. Actually, we have obtained those values based on a real H.264 flow, corresponding to DVB-SH standard requirements. We used pcap (packet capture) that consists of an application programming interface (API) for capturing network traffic. The pcap file has been generated by capturing the output of a video encoder, and it has been used to produce DVB traffic and thus the corresponding residual bandwidth. The resulting residual bandwidth varies between 9 and $15 \mathrm{kbits} / \mathrm{s}$ and has an average value of $13.5 \mathrm{kbits} / \mathrm{s}$, as shown in Figure 6).

On $3 \mathrm{G}$ network side, the data rate for services is 256

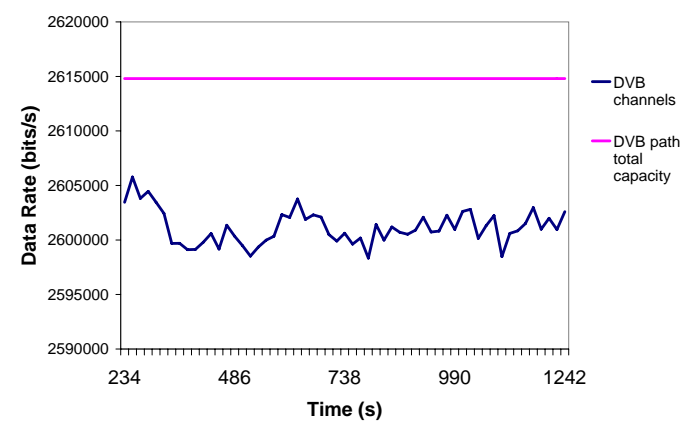

Figure 6: Residual bandwidth

$\mathrm{kbits} / \mathrm{s}$.

\subsection{Examples of scheduling algorithms}

Let us consider three 3G popular services S1, S2 and S3. $\mathrm{S} 1$ content size is $28 \mathrm{Mb}$. S2 content size is $23 \mathrm{Mb}$ and $\mathrm{S} 3$ content size is $18 \mathrm{Mb}$. Those contents correspond to short video flows of about one or two minutes duration. We assume that the three services accept the estimated waiting time. The planned order of service playing at terminal side is S1, S2 and finally S3. In a first simulation, the services are inserted over the residual bandwidth with respect to their planned playing order. Figure 7 shows that S1 starts being broadcasted over DVB-SH residual bandwidth immediately, then $\mathrm{S} 2$ starts being broadcasted at instant $\mathrm{t}=1080 \mathrm{sec}-$ onds, which means that Service S2 has waited 18 minutes before starting its delivery. $\mathrm{S} 3$ is started at $\mathrm{t}=2700$ seconds, i.e. after a waiting time of 45 minutes.

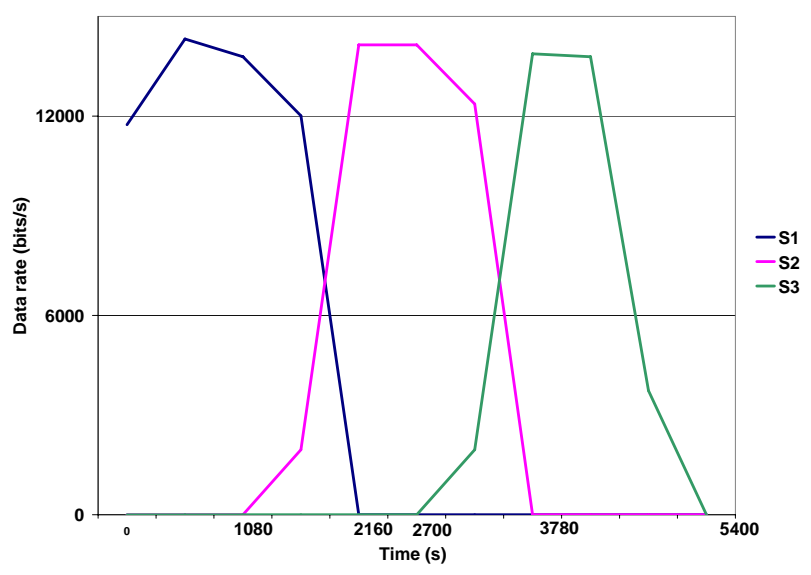

Figure 7: FIFO scheduling algorithm

Now, let us consider another scheduling algorithm. In this simulation, our objective is to maximize the number of popular 3G flows switched over the residual bandwidth. So, we privilege the transmission of the smallest flow. Figure 8

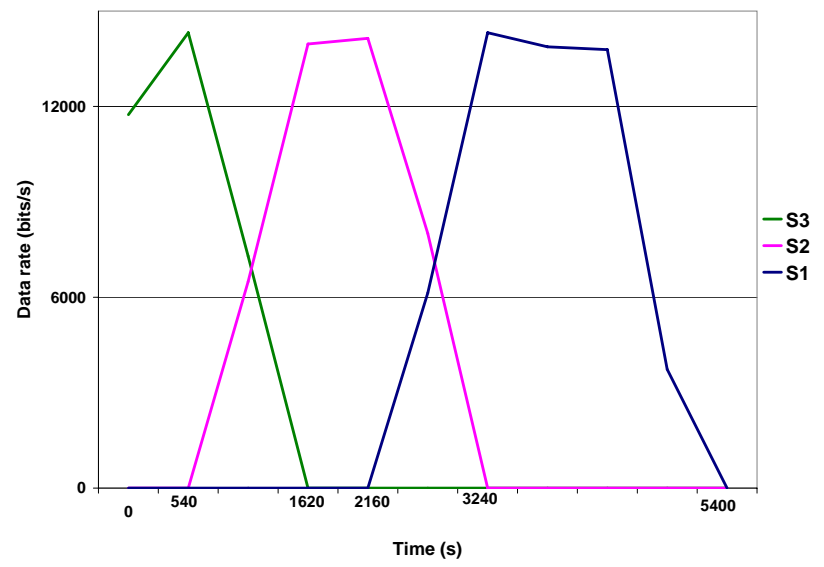

Figure 8: Smallest content first scheduling algorithm

shows that S3 is immediately inserted on the residual bandwidth as it is the one with the smallest content size. Second, $\mathrm{S} 2$ starts being transmitted at $\mathrm{t}=540$ seconds (i.e. after a 9 minute delay) and finally $\mathrm{S} 1$ starts being played at $\mathrm{t}=$ 2160 seconds (i.e. after a waiting delay of 36 minutes). We 
observe that the waiting delays have decreased. As a consequence, the probability for a service to accept the waiting time is greater. More services will accept being switched over DVB thus leading to a better use of $3 \mathrm{G}$ network capacities.

Delays may also be reduced if we anticipate flow transmission. Actually, terminals are equipped with caching memories where they can buffer their flows before playing. Many relevant scheduling policies are possible. In this study, we highlighted some of them. Depending on the targeted services, and on the operator objectives and priorities, there is a scheduling algorithm that best optimizes the use of the residual bandwidth in the considered case. We presented in this section relevant criteria to determine such an algorithm.

\section{EXTENSION OF THE DVB-SH RESIDUAL BANDWIDTH}

\subsection{Residual bandwidth extension}

A possible solution to reduce the transmission delays of $3 \mathrm{G}$ switched flows is to reserve some DVB-SH extra bandwidth. Actually, a 3G operator may pay for reserving some DVB channels, dedicated to switched $3 \mathrm{G}$ flows. For example, in France, the regulation authority specifies that a $120 \mathrm{kbits} / \mathrm{s}$ extra DVB bandwidth will be reserved for a future use. This use could be the design of complementary innovative services for Personal Mobile TV [12]. In this study, we consider that this additionnal bandwidth may be allocated for switching our $3 \mathrm{G}$ popular services.

This additive residual bandwidth will allow a considerable decrease of service transmission delay, thus enhancing users' quality of experience. We are now exploring the possibility to offer new services, thanks to this extended bandwidth.

We consider a bandwidth extension of $120 \mathrm{kbits} / \mathrm{s}$, which we add to DVB-SH default residual bandwidth (average value $13.5 \mathrm{kbits} / \mathrm{s}$ ). This bandwidth extension not only reduces the switched service delay, but it also allows the switching of some popular $3 \mathrm{G}$ real time services. Thanks to residual bandwidth extension, we are no more limited to the delivery of asynchronous services, with no real time constraints. We can now consider $3 \mathrm{G}$ real-time services with low data rates, like audio services for example. Such services may become popular, thus involving many unicast connections. The switching over DVB-SH network becomes an interesting alternative.

For our simulations, we consider an audio channel, addressed to a small user community and transmitted over 3G. Sometimes, this channel may provide very interesting programs, leading to a very important number of subscriptions. The service may be switched over DVB-SH during the transmission of the popular program, and then re-switch to a $3 \mathrm{G}$ multiple unicast transmission.

An important challenge here is to efficiently and dynamically manage the flow switching from one network to another, while maintaining service continuity. In this context, we chose to design a prediction algorithm that allows to anticipate users' behavior (i.e. subscriptions or unsubscriptions) and dynamically adapt our choice of delivery path to the predicted tendency. Our prediction algorithm is presented in the next paragraph.

\subsection{Prediction algorithm}

The provider decision to switch a flow to the DVB-SH path is mainly based on the number of subscriptions to the service. The $3 \mathrm{G}$ network decides to transmit this flow over the DVB-SH broadcast path as soon as the network switching criteria is fulfilled.

An idea is to consider a fixed value of subscriptions as a threshold beyond which the service is broadcasted over the residual bandwidth. Indeed, in the case of downloading popular files, users may often come and leave the network. This scenario will lead to switching between 3G and DVB networks each time the number of subscribers crosses the defined threshold value; the crossing can be in the higher or the lower direction. A fixed threshold value of subscriptions appears to be a bad approach.

We propose to use a prediction algorithm to perform a stable switch based on the users' subscriptions. The idea is based on studying the users' subscriptions between instants t0 and t1 in order to predict the potential new subscribers between instants $\mathrm{t} 1$ and $\mathrm{t} 2$. Then, based on this study, a decision is taken to switch the current network as shown in Figure 9. Hence, we implemented a prediction algorithm, based on the linear regression proposed in [13]. Moreover, we proposed to use two fixed thresholds according to the switching cost. When a very low number of users join the network, it may be worthwhile to keep the $3 \mathrm{G}$ channels even if a switch is advised by the prediction algorithm. This very low number of users is identified by what we call a low threshold ( 5 users in our simulations ).

When a very high number of users join the network, it may be worthwhile to keep using the DVB residual bandwidth even if a switch is advised by the prediction algorithm. This very high number of users is identified by what we call a high threshold (20 users in our simulations). The prediction algorithm is used between these two thresholds. In our sim-

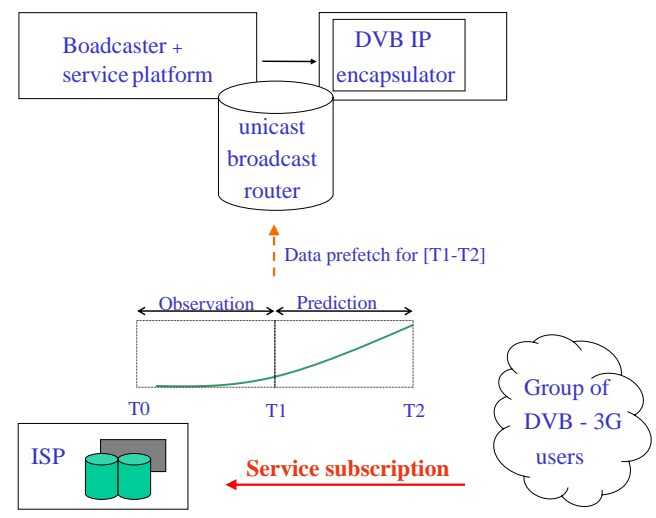

Figure 9: Subscription prediction

ulation, we consider a $120 \mathrm{kbits} / \mathrm{s} 3 \mathrm{G}$ flow. This could be, for example, a low quality audio video streaming flow. The simulation begins and users start subscribing to the service. The number of users increases rapidly. At $\mathrm{t}=13$ seconds, it crosses the low threshold number of users (5 users). As our algorithm predicts that the number of users will continue growing, the flow transmission is switched to the DVB-SH residual bandwidth, as shown in figure 10. The service be- 


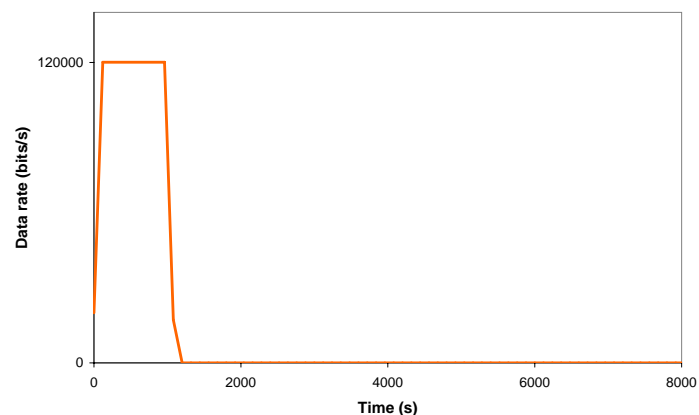

Figure 10: Service transmission over DVB

comes more and more popular, it continues taking benefit from DVB residual bandwidth broadcast capacities. The number of subscriptions still increases and crosses the high threshold (20 users). After a certain period of time, the service becomes less attractive, and users start unsubscribing. The number of users decreases and at $\mathrm{t}=1097$ seconds, it goes under the high threshold, as shown in figure 11. Our

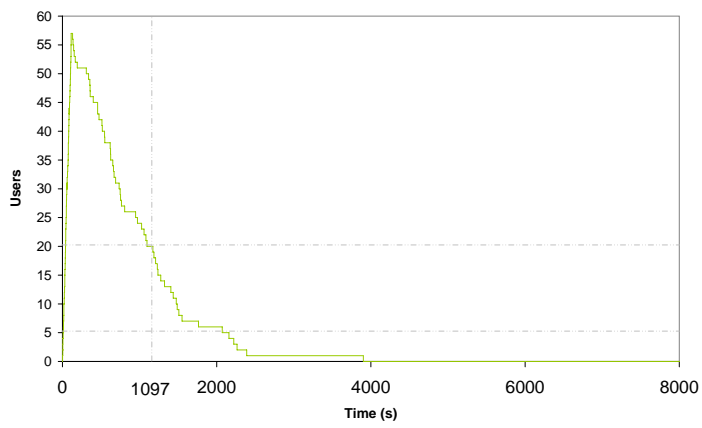

Figure 11: Number of subscriptions

algorithm predicts that the number of users will continue decreasing, so the service is switched back over $3 \mathrm{G}$ path, as shown in figure 12. Actually, it no more needs DVB broadcast capacities, so it will be delivered through $3 \mathrm{G}$ unicast connections. DVB residual bandwidth is now freed, another 3G popular service may use it to switch its contents.

In the example taken in the current section, our architecture supports the broadcast of a popular video flow at a low cost. In fact, the use of DVB eliminates the 3G unicast connections, and so optimizes the use of $3 \mathrm{G}$ radio resource. In a context where users can access more and more services on their mobile phones, such an optimization could be very interesting for service providers.

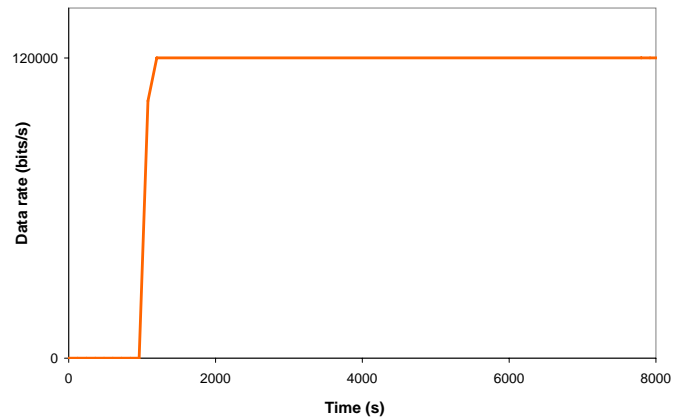

Figure 12: Service transmission over 3G

\section{CONCLUSION AND FUTURE WORK}

Our study permitted the definition of low cost services, over the initially unexploited DVB-SH residual bandwidth. Broadcasting some very popular $3 \mathrm{G}$ services over DVB residual bandwidth is an interesting alternative to the deployment of many expensive unicast connections. The introduced cost in terms of transmission delay is balanced by the low cost of subscription to such services. This work is a part of a large project that addresses inter-working a DVB-SH broadcast network with a third generation cellular network. DVB-SH brings very high broadcast capacities, while $3 \mathrm{G}$ networks provide interactivity, personalization and localization capacities, thanks to their upload link. We have used our proposed architecture and mainly the UBR router presence to realize an efficient switch of IP data into DVB networks.

Several proposals have been described to enable such a data switch while keeping a satisfactory user experience, and without disturbing DVB initial flows. Services that may benefit from the characteristics of such a network have been addressed. Scheduling algorithms to efficiently use DVB residual bandwidth have been proposed. And a user subscription prediction algorithm have been defined to dynamically switch from one network to another.

We are now studying the way to use Electronic Service Guide [14] messages to announce and manage our services. Our future work will concentrate on the design of other scenarios of services that benefit from the coupling between DVB-SH and $3 \mathrm{G}$ network.

\section{REFERENCES}

[1] A. Jedidi, M. Tlais, and F. Weis, "Coupling 3G with DVB networks for low cost services," International Conference on Engineering Management and Service Sciences, 2009.

[2] ETSI, "Digital video broadcasting (DVB); DVB specification for data broadcasting," European Standard EN 301 192, June 2004.

[3] EBU/ETSI, "Digital video broadcasting (DVB); implementation guidelines for the use of MPEG-2 systems, video and audio in satellite, cable and terrestrial broadcasting applications," European Standard ETR 154, September 1997. 
[4] ETSI, "Digital video broadcasting (DVB); framing structure, channel coding and modulation for digital terrestrial television," European Standard EN 300 744, November 2004.

[5] ETSI, "Digital video broadcasting (DVB);

transmission system for handheld terminals (DVB-H)," European Standard EN 302 304, November 2004.

[6] ETSI, "Digital video broadcasting (DVB); system specifications for satellite services to handheld devices (SH) below 3 GHZ," European Standard ETSI TS 102 585, 2008.

[7] J. Sesena, "Commonalities and peculiarities of DVB-S, DVB-C and DVB-SMATV systems (comm's and pec's of DVB systems)," International Broadcasting Convention, 1995.

[8] K. Schmidt, C. Gunter, and A. Rothermel, "Improving the mobility of DVB handheld devices with inter-carrier interference compensation," IEEE International Symposium on Consumer Electronics, 2004.

[9] "Ist-507312 fp6 european research project athena (digital switch over: Developing infrastructures for broadband access): http://www.ist-athena.org."

[10] D. Negru, "Convergence of IP and digital video broadcasting networks: from resource management to service provisioning," Ph.D. dissertation, UNIVERSITE DE VERSAILLES, Septembre 2006.

[11] "http://www.opnet.com."

[12] Conseil Superieur de L'audiovisuel, "Synthèse de la consultation sur le développement des services interactifs en télévision mobile personnelle."

[13] F. Leleu, "Techniques d'anticipation des acces à un service d'informations distribuees. application à un service de presse ecrite electronique," Ph.D. dissertation, Universite de Rennes I, 1997.

[14] DVB, "IP datacast over DVB-H : Electronic service guide (ESG)," DVB Document A099 Rev.1, September 2008 . 